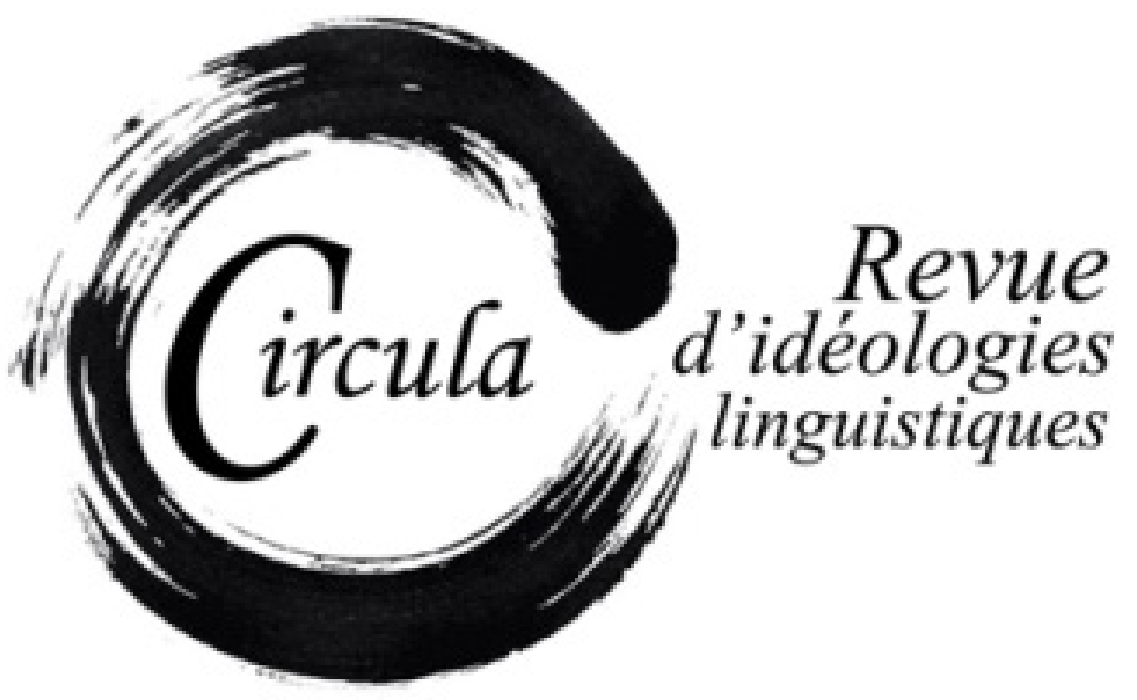

TITRE: DISSOCIER ALLOPHONIE ET VULNÉRABILITÉ : DE LA NÉCESSITÉ D'INTERROGER LES CATÉGORIES

Auteur: Maxime Alais, Université de Tours/UClouvain, Silvia LuCChini, UClouvain, Salima El

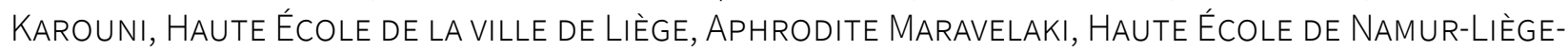
LUXEMBOURG, ÉLODIE OGER, UCLOUVAIN

Revue: Circula, NumÉro 12 : LA VULNÉRABILITÉ LINGUISTIQUE

DiRECTRICE: CLAUDIA TORRES CASTILLO

PAges: $87-106$

ISSN: 2369-6761

URI: HTTP://HDL.HANDLE.NET/11143/18444

DOI: HTTPS://DOI.ORG/10.17118/11143/18444 


\title{
Dissocier allophonie et vulnérabilité : de la nécessité d'interroger les catégories
}

\author{
Maxime Alais, Université de Tours/UCLouvain \\ maxime.alais@univ-tours.fr
}

Silvia Lucchini, UCLouvain silvia.lucchini@uclouvain.be

Salima El Karouni, Haute École de la ville de Liège salima.elkarouni @ hel.be

\begin{abstract}
Aphrodite Maravelaki, Haute École de Namur-Liège-Luxembourg afroditi.maravelaki@henallux.be
\end{abstract}

Élodie Oger, UCLouvain

elodie.oger@uclouvain.be

Résumé : Cet article s’interroge sur l'établissement des catégories d'élèves définies par les institutions éducatives belges francophones (Fédération Wallonie-Bruxelles, FWB) concernant la scolarisation des primoarrivants. Plus précisément, il s’agit de se demander si le lien établi par ces institutions ne crée pas un amalgame entre les élèves primoarrivants et ceux dits vulnérables, via un critère de non-maitrise supposée de la langue d'enseignement, ou du français langue d'apprentissage (FLA). Si les catégories définies par les autorités éducatives et les liens qu'elles établissent entre les élèves s'inscrivent dans un objectif global et louable d'aide à la scolarisation des élèves primoarrivants, nous considérons que ces catégories et liens qu'elles établissent viennent renforcer le regard négatif habituellement porté sur ce public scolaire et ne reconnaît pas suffisamment la diversité du public scolaire considéré dans son ensemble. Nous plaidons donc pour une dissociation entre l'allophonie et la vulnérabilité.

Mots-clés : Belgique francophone ; primoarrivants ; allophones ; regard déficitaire ; FLA 
Summary: This contribution questions the creation of categories of students as established by the French-speaking Belgian education authorities (Fédération Wallonie-Bruxelles, FWB) concerning the schooling of newcomers. More precisely, we are asking if the link established by these authorities doesn't create an amalgam between newcomer students and these students called vulnerable; they are supposedly linked together by the fact that they do not master the language of instruction, or French as the language of learning (français langue d'apprentissage, FLA). While these categories and the links they create between the students are based on a global and laudable objective of helping the schooling of newcomer students, we think that these categories and the links they create reinforce the negative perception people often have of this public and does not sufficiently recognise the diversity of the schooling public as a whole. We therefore argue for a dissociation between "allophones" and vulnerable students.

Keywords: French-speaking Belgium; newcomers; allophones; French as the language of learning 


\section{Introduction}

Depuis les années 1990, les pouvoirs publics belges francophones s'attachent à favoriser l'enseignement du français auprès des publics non francophones ou issus de l'immigration. Parmi les actions qui se sont multipliées en faveur de l'enseignement primaire ou secondaire, citons les cours de langues et de cultures d'origine (1996), présentés comme un tremplin pour une meilleure acquisition de la langue de scolarisation, les classes passerelles (2001) remplacées par les dispositifs d'accueil et de scolarisation des élèves primoarrivants (ou DASPA, 2012) ou, plus récents, les dispositifs de français langue d'apprentissage (FLA, 2019). Le développement de cours de FLE dans les hautes écoles (2001) et à l'université et de parcours d'intégration pour adultes primoarrivants non européens (2012) participent du même esprit. Pour une présentation de ces différentes mesures, on peut se référer à un article récemment publié par notre groupe (ALIA, 2018). Dans ce même article, nous défendions l'idée que si ces différentes mesures poursuivaient un objectif louable - favoriser l'intégration et la réussite scolaire/sociale des personnes immigrées ou issues de l'immigration -, elles avaient, dans certains cas, contribué à renforcer les processus de ségrégation à l'œuvre dans le système éducatif belge, qui, comme nous le savons depuis les enquêtes PISA, est l'un des plus inéquitables. Ces effets délétères ont du reste été cernés par de nombreux chercheurs en sciences de l'éducation qui ont porté leur attention plus généralement sur un public défavorisé et sur les mesures de discrimination positive (Merle, 2012). Le principal est probablement l'inégalité d'acquis, donnant un accès différencié aux diplômes et, par la suite, aux ressources socioéconomiques et sociétales.

\section{L’association hétéroattribuée entre allophonie et vulnérabilité}

On discerne presque toujours, à l'origine des mesures mentionnées, un espace de représentations au sein duquel les personnes issues de l'immigration sont perçues comme étrangères, non francophones ou parlant un français incorrect :

- Étranger. Même lorsqu'elles ont la nationalité belge, même après deux générations de permanence dans le pays de résidence. Nous avons montré dans une précédente étude qui portait sur la relation au français et aux autres langues de personnes issues de l'immigration de première, deuxième et troisième générations (Lucchini et al., 2012) que ce qui les qualifie d'étrangers, indépendamment de la nationalité, se rattache à des marques ethniques visibles. Un certain nombre d'informateurs interrogés dans la recherche en question estiment que l'apparence physique en particulier identifie la personne comme étrangère et est génératrice d'exclusion. À cause de la couleur d'abord, si celle-ci s'écarte d'un supposé phénotype prototypique local, caractérisé par « le blanc », «le blond » et « le bleu ». À ce propos, l'effet discriminant de la visibilité de la différence a depuis longtemps été mis en évidence (Beauchemin et al., 2010) et le « délit de sale gueule » est depuis longtemps dénoncé comme étant à l'origine de contrôles exagérés et abusifs. 
La caractéristique « étranger » est ensuite attribuée à partir du nom. En particulier du prénom. Une très intéressante étude de Prikhodkine et al. (2008) a montré que le prénom exolingue, parce qu'il traduit le choix explicite des parents, inscrit les individus dans la différence ethnique et est source de discrimination au travail en Suisse romande.

- Non francophone ou parlant un mauvais français, caractéristiques activées par la catégorie « étranger». Parfois l'attribution correspond à des faits. On peut en effet arriver dans le pays sans connaitre la langue qui y est parlée. Ou les enfants peuvent débuter leur scolarité en ayant entendu exclusivement une autre langue que le français dans leur milieu familial. Concernant la pratique d'un français non normé, on peut avoir appris le français en milieu scolaire exolingue, l'avoir peu pratiqué avant l'arrivée dans le pays d'accueil, sans l'avoir acquis de façon comparable à un natif. D'autant plus si le faible niveau de scolarité dans le pays d'origine, ou une absence de scolarité, n'a pas facilité l'analyse des différences et des ressemblances entre le français et la langue initialement parlée. Comme Klein (1989) le souligne, ce qui permet l'évolution d'une langue apprise en milieu naturel est la comparaison constante par le non-natif entre la langue qu'il produit et la production des natifs. L'acquisition s'arrête quand le non-natif n'est plus en mesure de percevoir des différences. Pour percevoir les différences, une analyse des deux langues est nécessaire. Et ce qui aide à le faire, ce sont les analyses explicites et parfois fastidieuses qu'on a effectuées au cours de la scolarité. Ces dernières ont en réalité contribué à former une sorte de grammaire intérieure de référence sur laquelle on se base pour comparer ${ }^{1}$.

Parfois l'association entre l'allophonie et la non-connaissance du français ou l'acquisition non normée du français se justifie lorsque les enfants ont grandi dans des contextes d'hybridation linguistique dans des quartiers populaires ethniquement homogènes, où la seule personne qui parle un français standard est finalement l'enseignant. Le phénomène est le même que celui qui a donné lieu à des pidgins ou à des créoles, où les deux langues en contact en ont produit une troisième possédant des caractéristiques des deux² (Lucchini, 2005). C'est donc cette langue partiellement composée de français et partiellement d'autres langues que les enfants ont entendue depuis leur plus jeune enfance et qu'ils ont apprise avant d'entrer à l'école, surtout si les contacts avec des natifs ont été réduits, ce qui est souvent le cas dans les quartiers ethniquement homogènes.

1. L'exemple de l'usage correct du participe présent italien est éclairant. Dans sa forme, le participe présent italien est différent du gérondif, contrairement au français, langue dans laquelle il est facile de les confondre. Les étudiants francophones utilisent en général le gérondif en italien à la place du participe présent car en français ils n’ont souvent pas appris à analyser correctement les deux et à les distinguer. Ils commettent donc l'erreur en italien à cause de cette défaillance d'analyse en français.

2. La différence entre les pidgins et la langue hybride parfois parlée dans les familles est que cette dernière est instable et fluctuante, sans doute à cause à la présence concomitante et constante des langues sources. 
Toutefois, la représentation des individus immigrés ou issus de l'immigration en tant qu'étrangers et des étrangers en tant que non francophones ou parlant un mauvais français peut être abusive et est le résultat d'hétéroattributions indûment généralisatrices. La confrontation avec une réalité contraire à la représentation ne la corrige pas. Le plus bel exemple est le dialogue entre l'enfant Girolamo Santocono, qui vit dans la Rue des Italiens, et Monsieur Léon, le marchand de fruit et légumes du coin :

De toute façon, comme il n'est resté presque exclusivement que les Italiens dans la rue, il a bien dû s'habituer. Et, dans un élan condescendant digne d'un touriste européen mettant la djellaba au cours de vacances en Tunisie, monsieur Léon a même appris notre langue. En fait, dans sa tête, il suffisait de mettre un « $i$ » ou un « a » au bout de chaque mot pour qu'à coup sûr tu parles le macaroni. (...)

Je me souviens qu'un jour, j'ai voulu tester sa bonne foi. J'ai rassemblé toutes les connaissances du bon parler français que j'avais acquises à l'école et je suis allé acheter des pommes de terre chez lui. Après son traditionnel « bon giorni » et mon très courtois « bonjour monsieur Léon », il m’a demandé:

- Tu volair queq'chosa, mon petito bambino?

- Oui, j'aimerais un sac de dix kilos de pommes de terre, répondis-je. Mais pas trop grosses car c'est pour cuire au four.

- Mucho bene! Mais pommas de terras novelas ou vieillas?

Dans son regard, je ne voyais aucune moquerie et je remarquais même qu'il faisait des efforts pour me comprendre. Tel un instituteur, il tendait l'oreille en avant à chacune de mes répliques. Je prends plaisir au jeu et je continue en exagérant.

- Oh, mon Dieu ! Mère ne m’a point donné de directives à ce sujet. Donnez m'en des plus récentes!

Son front s'est crispé brusquement. Manifestement, il ne comprenait plus.

- Quoi, toi volair novelas?

- Oui, exactement.

- Ah bon, parce que toi pas bene explicato, hein!

Il m’a servi ses meilleures pommes de terre et je suis reparti rassuré une bonne fois pour toutes. L'idée qu'un étranger puisse parler le français mieux que lui ne le traversait même pas et naturellement il a continué à me parler ainsi tout le temps. (Santocomo, 1987 : 126-127)

L'origine d'une telle surgénéralisation étranger-mauvais locuteur du français est à chercher, selon l'auteur de l'extrait, dans le sentiment éprouvé par la population locale de non-reconnaissance de la légitimité des immigrés italiens dans le quartier; un sentiment de perte de l'identité, de destruction de l'habitat et des relations sociales. Groupe importé contre personnes locales isolées, groupe qui impose sa loi et sa manière de vivre à des locaux qui n'ont finalement rien demandé et qui se sentent 
envahis. Identité collective contre identité individuelle, apparemment non conciliables. La puissance des hétéroattributions est à la mesure du sentiment d'expropriation vécue par les locaux, une façon de mettre à distance, d'empêcher ce qu'ils ont pu vivre comme une colonisation illégitime et définitive.

Ces catégories d'étranger, de non francophone ou de « parlant un mauvais français » ont été également hétéroattribuées par les institutions scolaires, qui sont en fin de compte tributaires des hommes qui les créent et de leurs représentations. Dans une publication de la Fédération WallonieBruxelles (l'instance qui administre l'enseignement francophone belge) parue en 2014 et destinée aux acteurs de l'éducation, la catégorie de non francophone disparait au profit de celle d' « allophone » (quelqu'un qui parle une langue autre que le français) et la catégorie de locuteur de français non standard en celle de «francophone vulnérable ». Le remplacement, en Belgique francophone, de la catégorie d'élève non francophone par celle d'élève allophone semble être un calque du même remplacement opéré en France en 2012 avec la parution d'une circulaire « relative à la scolarisation des élèves allophones nouvellement arrivés ${ }^{3} »$. Or, dans le contexte scolaire aussi, l'allophonie et la vulnérabilité peuvent être réelles, mais également imaginaires et abusives, et ces catégories peuvent générer des effets négatifs, que nous détaillons dans les pages qui suivent. Les quatre recherches que nous allons présenter s'inscrivent dans un programme plus vaste portant sur l'acquisition des langues en contexte migratoire ${ }^{4}$. Chaque recherche présentée possède une méthodologie propre, dont nous décrivons les grandes lignes, les détails étant spécifiés dans les publications citées.

\section{Un lien institutionnalisé entre élèves primoarrivants et élèves vulnérables}

La Fédération Wallonie-Bruxelles a promulgué trois décrets successifs relatifs à l'accueil des élèves primoarrivants. Le premier décret (2001) instituait les «classes-passerelles » qui deviendront dans le deuxième décret (2012) les « dispositifs d'accueil et de scolarisation des élèves primoarrivants » (DASPA). Très récemment (2019), un troisième décret reconduit le DASPA - moyennant des ajustements - et le complète aussi par la création d'un dispositif d'accompagnement FLA (Français langue d'apprentissage).

3. Information disponible sur_https://www.education.gouv.fr/bo/12/Hebdo37/MENE1234231C.htm_. [Page consultée le 20 août 2020.] À ce propos, Auger mentionnait en 2010 que la manière dont on nomme l'autre est signifiante, la dénomination «nouvellement arrivé » remplaçant primoarrivant à cause de l'association de cette dernière formulation avec primo-infectieux. Aussi, « nouvellement arrivé » est à préférer à « étranger » dans la mesure où la nouvelle dénomination indique que celui qui vient d'arriver est au début d'un processus d'intégration. La question qui toutefois s'ensuit est, comme le rappelle Auger, jusque quand est-on considéré comme «nouvellement arrivé ». Signalons que la même remarque peut être faite au sujet d'autres dénominations : jusque quand est-on qualifié d'allophone? Jusqu'à quelle génération est-on considéré comme « issu de l'immigration »?

4. Groupe de recherche ALIA, Acquisition des langues et immigration. 
Le décret DASPA-FLA (FWB, 2019) définit trois catégories d'apprenants :

(1) celle dite des «élèves primoarrivants ». Il s'agit d'élèves nouvellement arrivés sur le territoire belge, réfugiés ou candidats réfugiés, pour lesquels il y a une présomption de non-maitrise du français ${ }^{5}$, ou d'élèves en provenance de pays en voie de développement qui ne connaissent pas « suffisamment la langue de l'enseignement pour s'adapter avec succès aux activités de leur classe d'âge » (p. 2) ;

(2) celle des « assimilés aux élèves primoarrivants » qui désigne tout élève de nationalité étrangère, inscrit dans une école de la FWB depuis moins d'un an et dont la maitrise du français est jugée insuffisante suite à la passation d'une évaluation diagnostique ;

(3) celle dite des «élèves FLA-Français langue d'apprentissage » (pour les enseignements maternel et primaire). Il s'agit d'élèves francophones qui ne maitrisent pas suffisamment « la langue de l'enseignement pour s'adapter avec succès aux activités de sa (leur) classe d'âge » (p. 2). Leur maitrise insuffisante du français est, comme pour les élèves « assimilés aux primoarrivants », attestée par une évaluation diagnostique.

L'objectif de ce dernier décret est de " proposer un accompagnement scolaire et pédagogique adapté aux profils d'enseignement des élèves primo arrivants, assimilés et FLA et lié aux difficultés relatives à la maitrise de la langue de l'enseignement et de la culture scolaire » (p. 3). Dans ces trois cas de figure, il s'agit donc d'apprendre aux élèves la langue française et la culture scolaire.

Concernant les enfants primoarrivants, après des décennies d'invisibilité de la différence linguistique de départ, les décrets successifs à partir de 2001 ont introduit des dispositifs censés « voir la différence » pour l'assumer dans le contexte scolaire. En ce sens, on peut les considérer comme un progrès. On peut également considérer comme un progrès l'élaboration d'outils diagnostiques même si ceux qui accompagnaient le dispositif de la rentrée 2019 sont à améliorer, sans nul doute.

Dans l'article précédent (ALIA, 2018), nous mettions cependant l'accent sur le risque de ségrégation opéré par les catégorisations scolaires, qui isolent les élèves concernés par rapport aux autres, avec le risque de différencier leurs parcours par l'activation de représentations spécifiques. Dans ce cas, comme nous l'avons déjà évoqué par ailleurs, « voir la différence » reviendrait à « faire la différence ». En contexte éducatif français, Galligani (2012 : §4-5) souligne également la différenciation marquée entre le public primoarrivant et celui dit ordinaire, et l'homogénéisation réductrice que les catégorisations (liées au déplacement géographique, à la nationalité, à la maitrise de la langue notamment) opèrent sur la population scolaire concernée. Le public primoarrivant est diversifié, tant au niveau des origines ethniques et culturelles, des langues parlées que de la scolarisation (Maravelaki, 2007 ; Oger, 2019). Il n'est pas rare de voir placer par exemple des élèves arabophones dans des classes

5. Information disponible sur http://enseignement.be/index.php?page=28252\&navi=4593\&rank_page=28252. [Page consultée le 23 août 2020]. 
d'alphabétisation sous les prétextes parfois énoncés par les acteurs éducatifs qu'ils doivent " apprendre l'alphabet de notre langue » ou "se familiariser avec l'écriture alphabétique », souvent sans (se) demander si ces élèves ont été scolarisés, ont étudié ou parlent d’autres langues (ce qui est d'ailleurs souvent le cas).

De façon analogue, la reconnaissance du français en tant que langue d'apprentissage, le FLA, comme auparavant le français langue de scolarisation (le FLSCO), pourrait être considérée comme un progrès, dans le sens où l'on « verrait » enfin l'existence d'élèves pour lesquels le registre de la langue scolaire n'est pas absorbé dans le milieu familial. L'école se doit donc de l'enseigner sans le prendre pour acquis. Dans ce cas aussi cependant, « voir la différence » pourrait se transformer en «faire la différence », alors que tous les enfants doivent s'approprier cette langue d'apprentissage, chacun selon ses besoins, qui doivent être identifiés par les enseignants. Au lieu d'étendre à tous les élèves l'enseignement de la langue scolaire, fall ait-il créer une nouvelle catégorie (le FLA ne constitue pas une nouvelle langue), le FLA, abréviation devenue un acronyme qui désigne maintenant, décrétalement, des apprenants?

Le public scolaire est divers et, hormis le cas d'apprenants débutant en français, cette reconnaissance de la diversité doit se faire au sein d'une classe hétérogène pour qu'elle ne se transforme pas en ségrégation, en parcours scolaire de relégation en particulier, ce qui est par ailleurs visé par la recherche de la mixité sociale et ethnique qui active les décideurs politiques en Belgique depuis un certain temps. Si la reconnaissance de la diversité du public scolaire peut s'inscrire dans celui, plus vaste, de la problématique de la reconnaissance à l'échelle de la société (Honneth, 1992), nous estimons que les institutions éducatives doivent encore s'attacher à la construction d'un commun réfléchi par tous et pour tous. C'est à l'intérieur de ce commun que l'altérité linguistique et culturelle se doit d'être abordée, comme une normalité et non sur la base de catégories isolantes.

Enfin, l'association dans un même décret entre l'apprenant primoarrivant, supposé non francophone ou allophone, et les francophones supposés vulnérables pose question. La liaison retenue est que tous ont une « mauvaise ou la partielle compréhension de la langue dans laquelle les enseignements sont dispensés $»^{6}$, une «non-maitrise de la langue de l'enseignement», correspondant pour le décret au niveau B1 du Cadre européen commun de référence pour les langues (CECRL). Une mention particulière doit être faite pour le cas des élèves primoarrivants francophones. Les compétences de ces élèves sont souvent les plus dévalorisées dans le milieu scolaire. Leur « niveau de maitrise » pourrait être jugé insatisfaisant pour plusieurs raisons : accent déviant de la norme, système scolaire d'origine jugé inférieur au nôtre, difficultés à s'exprimer à l'oral ou à l'écrit dans des situations stressantes ou sous pression, par exemple, en situation de test de placement. Lors d'une recherche doctorale en cours (Maxime Alais), la direction d'une école qui scolarise des élèves primoarrivants francophones déclare : «ils sont francophones, mais il faut se méfier ». Si on y ajoute des facteurs

6. Propos extraits de l'allocution de la ministre de l'Éducation M.-M. Schyns lors de la journée d'information du 26 août 2019 concernant le décret DASPA-FLA : http://www.enseignement.be/index.php?page=28254\&navi=4594\&rank_ page=28254. [Page consultée le 2 août 2020.] 
indépendants de l'école, décrits ci-dessus, comme la couleur ou le nom, l'amalgame avec un public «vulnérable » et « allophone » s'opère sans difficulté et peut compromettre la présomption d'un parcours scolaire de réussite pour les primoarrivants.

\section{Une diminution des attentes scolaires}

Les conséquences des catégorisations qui procèdent par des distinctions qui ne sont pas toujours basées sur une analyse des besoins linguistiques réels, dans l'espace commun d'une classe socialement et ethniquement hétérogène, risquent de produire des effets délétères pour les populations concernées. En particulier, les exigences des formateurs à l'égard de ces publics sont nivelées par le bas, ce qui explique en partie la construction inégale des acquis scolaires que nous évoquions plus haut.

La recherche doctorale menée par Oger (2019) visait à identifier, à décrire et à analyser les pratiques pédagogiques des enseignants œuvrant dans les DASPA de l'enseignement secondaire belge francophone. En particulier, elle s'est attachée à comprendre les pratiques enseignantes susceptibles de favoriser l'intégration des élèves primoarrivants dans l'enseignement ordinaire.

Articulant approches quanti- et qualitative, le dispositif de recherche s'est déployé sur deux années scolaires et demie (de septembre 2015 à décembre 2018). Lors de la première phase, une quinzaine d'écoles organisant un DASPA ont été prélevées. Un test évaluant les compétences en lecture et en écriture a été soumis à trois reprises aux 600 élèves de l'échantillon, afin de mesurer l'évolution des élèves sur une année scolaire (2015-2016). C'est à l'appui des résultats émanant des tests que, lors d'une seconde phase, deux écoles ont été sélectionnées afin de servir de terrain à l'analyse qualitative. Les données qualitatives concernant ces deux DASPA ont été collectées au moyen des méthodes traditionnelles de l'ethnographie : afin d'accéder aux pratiques effectives, la chercheuse a été amenée à observer et à filmer pendant deux semaines des leçons de français, de mathématiques, d'histoire-géographie et de religion. Elle a également recueilli les documents distribués aux élèves lors de ces séances, ainsi que des documents plus généraux (grilles-horaires, document d'intentions pédagogiques, planification de matière). Les entretiens qui ont suivi avec les enseignants ont permis de recueillir leur point de vue sur les pratiques observées. D’autres interviews ont ensuite été réalisées avec les élèves de DASPA mais aussi avec les enseignants des classes ordinaires, la direction des deux écoles et les éducateurs. Les données recueillies sous la forme de vidéos et d'entretiens ont dans un premier temps été retranscrites, puis traitées à l'aide d'un synopsis (Schneuwly, Dolz et Ronveaux, 2006). Le repérage d'« épisodes critiques » de classe, c'est-à-dire des moments où surgissent des « obstacles » significatifs à l'enseignement et à l'apprentissage (Astolfi, 1992 ; Brousseau, 1983), a constitué le point de départ à l'analyse qualitative. Décrits puis analysés, ces obstacles ont, dans un dernier temps, été réinterprétés à la lumière des gestes professionnels (Bucheton et Soulé, 2009) que les enseignants, avec ou sans succès, mettent en œuvre pour les surmonter. 
L'analyse du matériau empirique a permis de mettre en évidence un écart de pratique important entre les deux écoles. Cette divergence de pratiques a pu être en partie expliquée par une série de facteurs, relevant notamment du champ des représentations sociales. Il est notamment apparu qu'un nombre important d'enseignants adhéraient à la représentation de l'étranger/allophone vulnérable. Ce rapprochement, en l'espèce, peut trouver à se justifier. Les enfants et les adolescents bénéficiant du dispositif sont de nationalité étrangère et répondent à un statut particulier (demandeurs d'asile, réfugiés, ressortissants d'un pays en voie de développement, ...). C'est d'ailleurs leur arrivée depuis moins d'un an en Belgique, parce qu'elle les trouve démunis face à la langue de l'enseignement, qui justifie leur présence dans le dispositif.

Cependant, par-delà les faits concrets qui la corroborent, cette représentation, lorsqu'elle est trop unanimement partagée par le corps enseignant, au point de la faire primer sur les objectifs d'enseignement et d'insertion scolaire, peut entrainer des répercussions dangereuses sur les plans pédagogique et didactique. Notamment parce que sont alors favorisées:

- l'adoption de postures professionnelles qui s'écartent de celle de professeur. Un certain nombre d'enseignants rencontrés se déclarent tantôt « psychologues », tantôt « assistants sociaux», «éducateurs », etc.

- la poursuite d'objectifs autres que scolaires. Certains de nos informateurs allouent au DASPA une visée purement « sociale », semblable à celles que poursuit l'enseignement de promotion sociale;

- une diminution des attentes scolaires à l'égard de ce public. Certains enseignants (autres que les professeurs de français) vont jusqu'à attribuer à leur cours une fonction strictement récréative.

Il n'est alors pas rare que cet amenuisement des exigences scolaires entraine l'adoption de pratiques enseignantes que nous avons appelées « simplificatrices » : des pratiques « réflexes » mais peu réflexives, et donc bien souvent stériles en ce qu'elles tendent à réduire la difficulté supposée des apprentissages sans que cette simplification n’aboutisse à un réel bénéfice didactique. Dans la gamme des pratiques simplificatrices les plus spontanément mises en œuvre, on retrouve: des interactions professeur-élèves restreintes et à la portée limitée, une utilisation d'expédients limitant l'usage du langage par l'élève (mimiques, réponses monosyllabiques ou asyntaxiques, « oui/non »), une éviction des opérations discursives complexes (telles que « définir », « justifier », « reformuler ») à la faveur d'opérations simples ou déictiques (nommer ou désigner, par exemple), une absence de progression marquée entre les activités proposées, une propension des enseignants à puiser dans les contenus les plus élémentaires de la discipline, une prédominance du format de l'exercice sur celui de la tâche, une propension à improviser la leçon d'un bout à l'autre, etc. En se systématisant, ces pratiques conduisent la plupart du temps à des situations tantôt adidactiques, tantôt très (trop) faiblement didactiques, qui rendent l'enseignement peu à même de faire progresser ces élèves. 
Ce sur-ajustement aux difficultés et caractéristiques des élèves est également l'un des résultats de l'enquête menée dans le cadre de la recherche doctorale de El Karouni $(2010,2012)$ au sein de deux classes de $5^{e}$ générale de l'enseignement secondaire. La chercheuse formulait ainsi l'hypothèse que les pratiques des enseignants sont influencées par leurs représentations des compétences et des pratiques langagières des élèves catégorisés comme issus de l'immigration, représentations qui sont notamment fondées sur leurs perceptions de leurs caractéristiques sociolinguistiques et ethniques. Elle a ainsi examiné comment les objectifs linguistiques sont définis et par quels moyens les enseignants vont tenter de les faire acquérir par leurs élèves. Cette dimension a été explorée à partir du dispositif méthodologique suivant (voir El Karouni, Collès et Lucchini, 2008 pour un descriptif détaillé). Dans la première phase de l'enquête de terrain, l'approche a été essentiellement quantitative et a concerné quatre classes de $5^{e}$ générale de l'enseignement secondaire (la moyenne d'âge des élèves est de 16 ans et demi), contrastées du point de vue socioethnique. Le champ d'observation a été réduit à deux classes fréquentées exclusivement par des élèves issus de l'immigration ${ }^{7}$ où les résultats obtenus par les élèves à une évaluation en langue ${ }^{8}$ sont les plus faibles et où les exigences linguistiques manifestées par les professeurs de français lors de cette passation sont contrastées ; l'une «surévalue » et l'autre «sous-évalue » les performances objectives de ses élèves. Ces postures de «tolérance » et de « sévérité » ont suscité des questionnements qui ont fait l'objet d'une analyse qualitative. L'exploration des questions suscitées par la première phase de l'enquête de terrain a été qualitative et examinée à travers des études de cas menées en parallèle dans les deux contextes. Plus précisément, les modalités d'ajustement aux caractéristiques ethniques et sociolinguistiques des élèves sont examinées à travers l'étude combinée des représentations et des pratiques enseignantes. Les données ont été recueillies dans les contextes naturels et émanent des questionnaires, des observations directes des pratiques d'enseignement et des entretiens semi-directifs conduits avec le professeur et les élèves.

El Karouni a ainsi montré comment la représentation des élèves comme «non francophones » avait des répercussions négatives dans les pratiques d'enseignement du français. L'information déduite de l'origine migratoire et nationale de l'élève est corrélée à une pratique linguistique donnée. Dit autrement, à l'origine nationale des élèves est associée une série de caractéristiques concernant les pratiques linguistiques.

L'hétérocatégorisation des élèves enclenche un mécanisme que l'on peut décrire comme suit. À partir de cette information (erronée en grande part), l'enseignante développe l'attente que ses élèves ne progresseront pas dans leur connaissance du code linguistique : cette prophétie se manifeste de manière directe à travers la quantité de travail exigée et produite (les pratiques d'évaluation sont

7. Ces deux classes appartiennent à des établissements inscrits en « discrimination positive », lesquels se caractérisent par l'appartenance des élèves à des quartiers défavorisés qui présentent un indice socioéconomique faible.

8. Une épreuve sur des savoirs linguistiques décontextualisés et la rédaction d'une lettre de demande argumentée adressée au bourgmestre ont été utilisées comme outils d'évaluation. Les lettres de demande argumentées ont été évaluées par trois binômes de correcteurs extérieurs sur la base d'une même grille d'évaluation et par les enseignantes de français de chacune des quatre classes selon leurs propres critères. 
axées sur les paliers primaires dans l'échelle des difficultés ; les démarches intellectuelles d'apprentissage sollicitées sont élémentaires ; les savoirs disciplinaires sont peu investis) mais aussi indirecte (l'analyse des interactions révèle des logiques d'accommodation contre-productives dans la construction de la norme) qui donnent peu d'occasions, pour les élèves, d'apprendre et d'améliorer leur performance en langue.

Un autre travers de cette fusion des données ethniques et sociolinguistiques dans les représentations réside dans la réification d'une catégorie ethnolinguistique qui n'est pas pertinente dans la triangulation didactique des apprentissages, et qui est justement celle de francophones vulnérables, apparaissant dans la brochure de la Fédération Wallonie-Bruxelles publiée en 2014 et déjà mentionnée. Comme nous l'avons développé par ailleurs (El Karouni, 2017), ces derniers, peut-on y lire, «se perçoivent souvent comme Belges francophones. Certains d'entre eux ont été primo arrivants et scolarisés en classe passerelle ou en DASPA, d'autres appartiennent aux $2^{\mathrm{e}}$ ou $3^{\mathrm{e}}$, voire $4^{\mathrm{e}}$ ou $5^{\mathrm{e}}$ génération de l'immigration, d'autres encore sont d'origine belge » (p. 7). Les élèves sont caractérisés dans un registre plus subjectif qu'objectif: on ne dit pas de ces élèves qu'ils sont «Belges francophones » mais qu'ils «se perçoivent » comme tels. Le curseur n'est pas placé sur le statut administratif - être de nationalité belge est un fait objectivable - mais sur l'adéquation - moins objectivable car placée sur le plan de la perception - aux caractéristiques du « Belge francophone » qui fonctionne à cet endroit comme une catégorie ethnolinguistique.

Ces observations plaident, nous semble-t-il, pour que dans les discours la nature des besoins linguistiques en termes d'apprentissage soient dissociées de l'origine nationale des élèves. Ce changement de discours peut être soutenu par la pratique systématique d'une évaluation de type diagnostic à propos des ressources linguistiques disponibles et des besoins en langue.

\section{L'affaiblissement du sentiment d'appartenance à la langue com- mune et plus largement au pays de résidence}

Les catégorisations n'ont pas seulement un effet sur le parcours scolaire et l'acquisition linguistique. Elles ont aussi un effet sur la cohésion sociale des composantes ethniques de la société. Le sentiment d'appartenance à la langue commune et plus largement au pays de résidence (Lucchini et al., 2008) ressort affaibli par l'attribution abusive d'une incompétence linguistique sur la base de marqueurs ethniques tels que la couleur ou le nom, même chez les personnes nées et scolarisées en Belgique francophone.

Dans la recherche menée à la fin des années 2000 et mentionnée supra, nous interrogions le rapport au français de la population issue de l'immigration de la première, deuxième et troisième génération. Ont été interrogées 65 personnes originaires de 19 pays (39 à Bruxelles et 24 dans la partie francophone du pays) ; 22 de première, 43 de deuxième et 5 de troisième génération ; 33 hommes et 32 femmes ; 41 personnes âgées entre 30 et 49 ans, 19 de moins de 30 ans et 5 de plus de $50 ; 46$ 
diplômés de l'enseignement secondaire au moins. La recherche visait à décrire les pratiques linguistiques et à comprendre le système des représentations qui portent sur ces pratiques (statuts, valeurs, caractéristiques attribués aux langues, sentiment d'appartenance aux communautés, affects reliés à la pratique des langues, etc.). Les entretiens semi-dirigés ont été menés à partir d'un guide qui comprenait sept volets visant à décrire a) les parcours migratoires, b) les pratiques linguistiques, les statuts des langues parlées, les valeurs qui y sont rattachées et les motivations sous-jacentes, c) les usages et les souhaits concernant les « langues d'origine » (nous avons choisi d'utiliser cette dénomination dans les entretiens), d) la perception du plurilinguisme, e) le sentiment d'appropriation de la langue française, f) la question de la norme linguistique, g) les rapports entre les langues et la réussite scolaire et professionnelle.

L'association hétéroattribuée entre minorité visible, étranger et mauvaise connaissance du français a été soulignée par plusieurs informateurs comme étant à l'origine de pratiques de discrimination indirectes, générées par ces représentations négatives d'une non-correspondance au prototype «blanc-bleu-blond ». Ces pratiques de discrimination ont par ailleurs été corroborées par une série d'études, en Belgique ou non (v. le rapport de l'OCDE de 2008, qui conclut à une inégalité importante entre la population immigrée ou d'origine immigrée en ce qui concerne l'accès à l'emploi, en particulier pour les femmes, et également pour les générations scolarisées en Belgique ; v. également la recherche déjà citée menée en Suisse romande [Prikhodkine et al., 2008]).

Ce que la recherche mentionnée a laissé émerger, c'est que les personnes qui subissent ces pratiques de discrimination vont chercher à se former une image d'eux-mêmes cohérente et positive en récupérant d'autres marques ethniques. Parmi celles-ci, les langues associées à l'origine. Elles vont être réactivées ou apprises, qu'elles aient été ou non parlées par des ancêtres parce qu'elles vont permettre la reconnaissance réciproque à un groupe humain, accueillant cette fois, et vaste. Pour cette raison les langues standard, pouvant être transnationales, vont être privilégiées. Ce sont ces langues qui vont faire l'objet d'un investissement affectif.

A contrario, il apparait clairement que ce processus a comme conséquence un affaiblissement du sentiment d'appartenance à la société de résidence et à sa langue. Cela produit un désinvestissement affectif par rapport à la langue française : cette population, immigrée ou issue de l'immigration, se voit en quelque sorte expropriée du français. Au point que des personnes peuvent hésiter à se définir francophones, même quand le français est la seule langue qu'ils parlent. La valence affective attribuée aux langues d'une origine proche ou lointaine est niée au français, qui devient une langue nécessaire et utilitaire, comme langue de l'espace public, et qui en quelque sorte s'affaiblit. Dans tous ces cas, le regard qui caractérise l'autre comme un « allophone » et « vulnérable » finit par produire la vulnérabilité attribuée, en termes d'acquisition de la langue et de désinvestissement affectif. 


\section{Dissocier allophonie et vulnérabilité}

L'association entre allophonie et vulnérabilité replace le focus sur les manquements, les déficits ou les « handicaps » des élèves primoarrivants au lieu de se concentrer sur le renforcement des compétences ou des aptitudes déjà existantes.

En cela, il est à regretter que les outils de travail très concrets fournis par le Conseil de l'Europe, à travers sa politique linguistique et la publication du CECRL, soient très peu exploités par les écoles. Nous retiendrons ici deux concepts particulièrement intéressants : la définition positive de la compétence (CECRL, 2001) ainsi que le plurilinguisme et l'éducation plurilingue?.

En effet, dans le CECRL, la définition des compétences est toujours positive. Les niveaux sont définis par rapport à ce que l'apprenant est capable de faire et non pas à ce qui est attendu (norme) ou à ce qu'il n'est pas encore en mesure de faire (maitrise/non maitrise). Il accepte que les compétences puissent être partielles et il laisse la place au développement d'interlangues, ce qui nous permet de travailler différemment en classe et de mieux évaluer les acquis. Une application mécanique et artificielle des niveaux ou des compétences attendues pour, par exemple, avoir la nationalité (A2) ou pour intégrer une classe « ordinaire » (B1) trahit cet esprit et ne donne pas les résultats souvent souhaités par les enseignants, c'est-à-dire des classes homogènes sur le plan de la « maitrise de la langue ».

Même si, sur le plan didactique, le CECRL (2001) peut s'avérer très intéressant, le concept du plurilinguisme et de l'éducation plurilingue offre un changement de vision et de paradigme, d'une école unilingue et uniculturelle qui peine à enseigner même une deuxième langue, à une école où toutes les langues sont possibles. La compétence plurilingue comprend toutes les langues et les variétés linguistiques du répertoire d'un individu. Ces compétences sont de nature et de niveau différents seIon les langues au sein de son répertoire allant du balbutiement de quelques mots dans une langue à un contrôle quasi total d'une autre. La compétence plurilingue dépend des contextes d'utilisation et des besoins langagiers des usagers. Dans ce sens, un individu pourrait faire des études en ingénierie dans une langue sans nécessairement pouvoir tenir une conversation « sociale» dans cette langue.

L'éducation plurilingue, selon Beacco et Byram (2007), encourage la prise de conscience du pourquoi et du comment on apprend les langues cibles; la prise de conscience de compétences transposables et de la capacité à les réutiliser dans l'apprentissage des langues ; le respect du plurilinguisme d'autrui et de la reconnaissance des langues et de leurs variétés, quelle que soit l'image qu'elles ont dans la société ; le respect des cultures inhérentes aux langues et de l'identité culturelle d'autrui ; la capacité à percevoir et à assurer le lien entre les langues et les cultures; une approche intégrée de l'enseignement des langues dans les curricula.

9. Information disponible sur www.coe.int/lang/fr. [Page consultée le 30 mars 2021.] 
Il nous parait évident que ce changement de paradigme dans les écoles ne sera pas possible sans une rupture dans la formation, initiale et continue, des enseignants. Afin d'y arriver, une approche intégrée est nécessaire non seulement pour l'enseignement des langues mais aussi dans les curricula des formations universitaires et supérieures.

Le plurilinguisme et l'éducation plurilingue sont des concepts qui peuvent certainement soutenir un regard positif sur la diversité linguistique et culturelle. Mais pour dissocier davantage le public primoarrivant et d'autres publics en difficulté scolaire, et l'allophonie de la vulnérabilité, une recherche en cours déjà mentionnée (Maxime Alais) développe de nouvelles mises en liens entre le public primoarrivant scolarisé en FWB et d'autres publics qui doivent apprendre une nouvelle langue de l'école, notamment celui du réseau AEFE (Agence pour l'enseignement français à l'étranger), en mettant l'accent sur le concept d'altérité linguistique et culturelle, et non plus sur la notion d'allophonie seule, puisqu'elle est employée pour séparer, dans les discours et dans les pratiques, certains élèves du public scolaire considéré dans son ensemble. Pour Auger (2019) également, d'ailleurs, l'emploi de la notion d'allophonie témoigne d'un refus de reconnaissance du plurilinguisme et de l'expertise des élèves primoarrivants. La recherche décrite ci-après tente de penser autrement l'altérité linguistique et culturelle des élèves que sous le prisme seul de la notion d'allophonie.

Le regard usuel porté sur la scolarisation des apprenants primoarrivants en FWB est largement problématique : il insiste sur des caractères déficitaires et (très) spécifiques, a minima non ordinaires de ce public. Or, ce regard déficitaire assigne et homogénéise ce public scolaire, pourtant fondamentalement pluriel. Pour renouveler ce regard qui assigne l'autre, le choix de cette recherche en cours (et son originalité) a été de parier sur la comparaison entre la situation de DASPA en FWB et celles d'écoles appartenant aux réseaux AEFE ou CIS (Council of International Schools). Ces deux dernières situations sont habituellement considérées disjointes ou très différentes de celle qui concerne la scolarisation d'apprenants primoarrivants. Pourtant, elles ont un point commun majeur avec celle-ci : la langue de l'école y est, pour une majorité de leurs apprenants, une langue étrangère/seconde. Or, cette scolarisation dans une autre langue semble, dans ces deux situations de comparaison, présentée et/ou vécue de manière beaucoup plus positive par les acteurs éducatifs et par les apprenants.

Nous avons donc dans un premier temps comparé des discours officiels issus des trois situations retenues, puis effectué des entretiens et des observations dans quatre écoles : deux écoles belges francophones bénéficiant d'un DASPA, une école du réseau AEFE située en Asie de l'Est et une école du réseau CIS située en Belgique. Concernant l'interprétation des discours, des extraits de ceux-ci ont été sélectionnés après un relevé d'occurrences de termes-clés, puis comparés. Des entretiens compréhensifs (Kaufmann, 2014) ont ensuite été menés dans les quatre écoles retenues avec les directions des écoles, des enseignants et des apprenants, ainsi que des observations en situation (Martineau, 2005). Pendant les entretiens, des extraits de discours sélectionnés après relevés d'occurrence ont notamment été utilisés comme déclencheurs de discussion. Pour l'interprétation des discours, la passation des entretiens et les observations effectuées, nous avons mis à profit notre expérience professionnelle personnelle d'enseignant de français dans un des deux DASPA retenu, 
en « Assum[ant] la recherche comme teintée dans la masse par la présence et l'expérience des chercheurs » (Huver, 2014:23; voir aussi Castellotti, 2017 : 10). Par ailleurs, notre démarche comparatiste s'inscrit dans la perspective herméneutique telle que défendue par Detienne (2000) et par Jucquois (2000). Pour celui-ci,

Le comparatisme instaure l'obligation d'une multiplicité de regards sur un objet supposé identique. Il débouche sur une prise de conscience de la complexité, propriété qui, lorsqu'elle concerne l'homme, entraîne une perception plus aigüe de la diversité et du nécessaire pluralisme qui en découle. (Jucquois, $2000: 18-19)$

La comparaison/le croisement entre des terrains et des publics scolaires usuellement considérés disjoints (les primoarrivants «migrants » associés aux « vulnérables » d'un côté, les riches « expatriés » et «natifs » privilégiés de l'autre) renouvelle les discours déficitaires habituellement tenus à propos des apprenants primoarrivants et éclaire une problématique de reconnaissance de l'altérité linguistique et culturelle. Par ailleurs, confrontés à des discours institutionnels bien davantage positivants face à la diversité/l'altérité linguistique et culturelle, les acteurs de terrain développent un regard beaucoup moins négatif/déficitaire sur la scolarisation d'apprenants dits allophones.

Mais les observations effectuées sur un terrain scolaire AEFE montrent qu'au-delà des discours communicants/promotionnels tenus par les institutions, les apprenants qui doivent apprendre une nouvelle langue de l'école échappent rarement à une mise en altérité, quel que soit le contexte scolaire. Ces différentes situations/institutions scolaires gagneraient donc à entrer en relation, à échanger, à développer des partenariats, etc., pour vivifier les discours qu'elles tiennent à propos des élèves qui doivent apprendre une nouvelle langue de l'école, pour diversifier leurs pratiques didactiques; pour interroger cette mise en altérité souvent observée.

Enfin, cette recherche met en évidence un partage de la problématique de la reconnaissance de l'altérité qui dépasse largement le seul critère de la langue « autre » comme la notion d'allophonie peut le laisser entendre. La notion d'allophonie n'est-elle pas une réduction de la notion d'altérité ? L'altérité linguistique et culturelle n'est-elle pas unanimement partagée entre nous tous, même lorsque nous parlons la même langue ? Plutôt que de la réserver aux " autres », ne faudrait-il pas mettre en avant son partage entre tous? En ce sens, l'école n'est-elle pas elle aussi allophone face aux apprenants visés par les dispositifs mentionnés? Autres problématiques que nous approfondirons dans un autre article. 


\section{Références}

Acquisition des langues et immigration (ALIA) (2018), "Politiques linguistiques dans les contextes multilingues de la Belgique francophone : aide ou ségrégation ? », Synergies Pays germanophones, n 11, p. 185-197.

Astolfi, Jean-Pierre (1992), «Apprendre parfranchissement d’obstacles ? », dans Hélène Romian (dir.), Repères, recherches en didactique du français langue maternelle, n5, p. 103-116.

Auger, Nathalie (2010), Élèves nouvellement arrivés en France, Paris, Éditions des archives contemporaines.

Auger, Nathalie (2019), "Allophone” : trajectoire d'une catégorie aux prises avec la notion d'expertise », TRANEL : Travaux neuchâtelois de linguistique, vol. 70 (L'expertise dans le champ des sciences du langage sur des terrains sensibles: un point de vue épistémologique, sous la dir. de Nathalie Auger et Maud Verdier), p. 23-41.

Beauchemin, Cris, Christelle Hamel, Maud Lesné, Patrick Simon et al. (2010), « Les discriminations : une question de minorités visibles », Populations et Sociétés, n 466.

Beacco, Jean-Claude et Michael Byram (2007), De la diversité linguistique à l'éducation plurilingue: guide pour l'élaboration des politiques linguistiques éducatives en Europe, disponible sur www.coe.int/fr/web/platform-plurilingual-intercultural-language-education/three-fundamental-and-complementary-tools-for-the-implementation-of-plurilingual-education. [Page consultée le 20 août 2020.]

Brousseau, Guy (1983), "Les obstacles épistémologiques et les problèmes en mathématiques », Recherche en didactique des mathématiques, vol. 4, n² 2, p. 164-198.

Bucheton, Dominique et Yves Soulé (2009), «Les gestes professionnels et le jeu des postures de l'enseignant dans la classe : un multi-agenda de préoccupations enchâssées », Éducation et didactique, vol. 3, n³, p. 29-48.

Castellotti, Véronique (2017), Pour une didactique de l'appropriation : diversité, compréhension, relation, Paris, Didier.

Conseil de l'Europe (2001), Cadre européen commun de référence pour les langues : apprendre, enseigner, évaluer, disponible sur www.coe.int/fr/web/common-european-framework-reference-languages. [Page consultée le 20 août 2020.]

Detienne, Marcel (2000), Comparerl'incomparable: oser expérimenter et construire, Paris, Seuil.

El Karouni, Salima (2012), «L'enseignement du français en contextes scolaires hétérogènes : pour un renouvellement des repères didactiques traditionnels », Recherches et Éducations, nº 7, p. 149165.

El Karouni, Salima (2017), «La place de l'ethnicité au sein du champ de l'enseignement/apprentissage du français », Études en didactique des langues, n²8, p. 59-80. 
Fédération Wallonie-Bruxelles (2014), Enseigner aux élèves qui ne maitrisent pas la langue d'enseignement, disponible sur http://www.enseignement.be/index.php?page=0\&navi=3627. [Page consultée le 20 août 2020.]

Fédération Wallonie-Bruxelles (2019), Décret visant à l'accueil, la scolarisation et l'accompagnement des élèves qui ne maitrisent pas la langue dans l'enseignement organisé ou subventionné par la Communauté française, disponible sur https://www.gallilex.cfwb.be/document/ pdf/46275_000.pdf. [Page consultée le 20 août 2020.]

Galligani, Stéphanie (2012), « Regards croisés sur les enfants venus d'ailleurs et scolarisés en France », Les Cahiers du GEPE, $n^{\circ} 4$ (Les langues des enfants «issus de l'immigration» dans le champ éducatif français), disponible sur http://cahiersdugepe.fr/index.php?id=2314\#texte. [Page consultée le 25 octobre 2020.]

Honneth, Axel (1992), La lutte pour la reconnaissance, Paris, Gallimard.

Huver, Emmanuelle (2015), « Prendre la diversité au sérieux en didactique/didactologie des langues. Altériser, instabiliser: quels enjeux pour la recherche et l'intervention? 》, dans Emmanuelle Huver et David Bel (dir.), Prendre la diversité au sérieux en didactique/didactologie des langues. Altériser, instabiliser: quels enjeux pour la recherche et l'intervention? Paris, L'Harmattan, p. 11-24.

Jucquois, Guy (2000), "Le comparatisme, éléments pour une théorie », dans Guy Jucquois et Christophe Vielle (dir.), Le comparatisme dans les sciences de l'homme : approches pluridisciplinaires, Bruxelles, De Boeck, p. 17-46.

Kaufmann, Jean-Claude (2014), L'entretien compréhensif, Paris, Armand Colin.

Klein, Wolfgang (1989), L'acquisition de langue étrangère, Paris, Armand Colin.

Lucchini, Silvia (2005), «L'enfant entre plusieurs langues : à la recherche d'une langue de référence », Enfance, vol. 57, n 4, p. 299-317.

Lucchini, Silvia (2012), « De la langue à la cohésion sociale ou de la cohésion sociale aux langues?», dans Virginie Conti, Jean-François De Pietro et Marinette Matthey (dir.), Langue et cohésion sociale : enjeux politiques et réponses de terrain, Neuchâtel, Délégation à la langue française, p. 87110.

Lucchini, Silvia, Philippe Hambye, Gilles Forlot et Isabelle Delcourt (2008), «Francophones et plurilingues: le rapport au français et au plurilinguisme des Belges issus de l'immigration », Français et société, no 19, p. 1-90.

Maravelaki, Aphrodite (2007), «Les classes-passerelles en Belgique francophone : vers une transformation de l'école uni-culturelle? », dans Silvia Lucchini et Aphrodite Maravelaki (dir.), Langue scolaire, diversité linguistique et interculturalité, Cortil-Wodon, Éditions modulaires européennes, p. 195-212.

Martineau, Stéphane (2005), «L'observation en situation : enjeux, possibilités et limites », Recherches qualitatives, hors-série $n^{\circ} 2$ (Actes du colloque "L'instrumentation dans la collecte des données 》 tenu à l'Université du Québec à Trois-Rivières le 26 novembre 2004), p.5-17. 
Merle, Pierre (2012), La ségrégation scolaire, Paris, La Découverte.

OCDE (2008), Les migrants et l'emploi, vol. 2 : l'intégration sur le marché du travail en Belgique, en France, aux Pays-Bas et au Portugal, disponible sur www.oecd.org/fr/belgique/lesmigrantsetlemploivol2lintegrationsurlemarchedutravailenbelgiqueenfranceauxpays-basetauportugal. htm. [Page consultée le 25 août 2020.]

Oger, Élodie (2019), Faire-passerelle : analyse systémique des pratiques enseignantes dans les dispositifs d'accueil et de scolarisation des élèves primoarrivants de Belgique francophone, thèse de doctorat, Louvain-la-Neuve, Université catholique de Louvain.

Prikhodkine, Alexei, Pascal Singy, Noémie Charton et Alessandro lannelli (dir.) (2008), « Tout nom n'est pas bon à dire : discriminations à l'embauche en Suisse romande », numéro thématique du Bulletin de linguistique et des sciences du langage, nº 24.

Santocono, Girolamo (1987), Rue des Italiens, Mons, Éditions du Cérisier.

Schneuwly, Bernard, Joaquim Dolz et Christophe Ronveaux (2006), « Le synopsis : un outil pour analyser les objets enseignés », dans Marie-Jeanne Perrin-Glorian et Yves Reuter (dir.), Les méthodes de recherche en didactiques : actes du premier séminaire international sur les méthodes de recherches en didactiques de juin 2005, Villeneuve d'Asca, Presses universitaires du Septentrion, p. 175-189. 